www.conferenceie.ase.ro

\title{
MULTI-CLASS TEXT SUPERVISED CLASSIFICATION ON ROMANIAN FINANCIAL BANKING REVIEWS
}

\author{
Irina RAICU \\ Bucharest University of Economic Studies, Romania \\ irina.raicu@ie.ase.ro \\ Răzvan BOLOGA \\ Bucharest University of Economic Studies, Romania \\ razvanbologa@ase.ro \\ Radu CONSTANTINESCU \\ Bucharest University of Economic Studies, Romania \\ radu.constantinescu@ie.ase.ro
}

\begin{abstract}
Nowadays, with the proliferation of social networks, forum discussions, blogs, and other forms of expression, more and more users have engaged in financial banking communities to gather information about financial banking products or services, to share experiences or to interact with other users. Reviews expressed by users on social media and Internet search activity are a valuable source of information. Text Classification is a growing research area used in social media analysis. The usefulness of Text Classification is manifested in different areas for various needs. For instance, the ability to automatically classify a review into its right financial banking product or service category would be appreciated by users when looking for a specific product or service as well as financial banking institutions to analyze the customers' feedback. Most of the research papers are focusing on exploring Text Classification methods for English-based text. Further, supervised machine learning methods for Text Classification require a huge data volume. In this paper, we focus on implementing supervised machine learning methods for multi-label text classification of Romanian financial banking reviews. We investigate the efficiency of using various supervised machine learning algorithms such as Random Forest, Support Vector Machine, Multinomial Nä̈ve Bayes and Logistic Regression to classify text applied to a limited amount of Romanian financial banking reviews. We analyze the corpus, features and the results data. The baseline features are unigrams and bigrams weighted by TF-IDF. The results illustrate that the SVM classifier outperforms in classification of text into 30 categories of financial banking products and services from Romanian reviews.
\end{abstract}

Keywords: Machine Learning, Romanian Reviews, Multi-Class Text Classification, Financial Banking Social Media

JEL classification: C3, C4, L86

DOI: $10.12948 / \mathrm{ie} 2019.01 .06$

\section{Introduction}

The fast growth of social media is constantly increasing online communication in financial banking domain embracing customers' digital behavior. Customers are using powerful channels in order to express their thoughts, sentiments, ideas and feelings about a financial banking product or service. Thus, this large number of customers' reviews or suggestions represents useful and valuable information. Text classification is an active research area widely explored by many researchers. The main goal of text classification methods and techniques is 
to classify a huge number of documents into a set of predefined categories based on its content. [1][2] Customer reviews or opinions are often short text documents which can be mined to determine useful information from the review. [3] Due to the diversity of information in text documents, the classification is not an easy process. Therefore, developing algorithms that can handle and develop text classification and increase its efficiency are still highly required. Most of the text classification methods and techniques are designed to handle English documents. Few researchers explore Romanian text classification methods and techniques. [4] Supervised machine learning methods for text classification require a huge amount of textual data. This research paper explores various supervised machine learning methods such as Linear Support Vector Classification, Logistic Regression, Multinomial Naïve Bayes, and Random Forest for multi-class text classification of Romanian limited amount of reviews. We represent the textual information as a vector space model (VSM). We incorporate unigrams and bigrams based on Term Frequency Inverse Document Frequency (TF-IDF) weighted method. As feature selection method, Chi square and document frequency is used.

\section{Multi-Class Text Classification}

Multi-class text classification is a supervised-learning process which classify the documents in given classes. Usually, multi-class classification methods consist of two phases: learning and classification. [5] In the learning phase, a training set where all documents are already labeled is used. The classifier learns from the training set and builds a model. In classification phase, the classification model is used to correctly classify a new unlabeled document. The Text Classification process is divided into four main steps: Data collection and preprocessing, building the model (feature selection), model evaluation and model testing (classification of new documents with unknown label classes) [6] detailed in the following sub-sections.

\subsection{Data collection and preprocessing}

The data available on the Web consists predominantly of unstructured text. Using web scraping technique to perform automated and targeted extraction of data, a dataset composed by $\mathbf{2 2 3 4}$ of posts from Conso portal (www.conso.ro) from the Romanian financial banking marketplace has been created. The dataset is detailed in [7]. The financial banking products and services are classified into 30 categories as shown in Figure 1. Each post from Conso contains a label with the financial banking product or service that the customers refer to. The aim is to classify the text document from the dataset into one of these 30 categories in order to identify automatically the financial banking product or service. As expected, we have a dataset with imbalanced class distribution; therefore, the identification performance on the small class is usually unsatisfactory. To overcome this limitation, we must balance the abilities between the classes. Thus, we have under sampling all the classes to 100 of values.

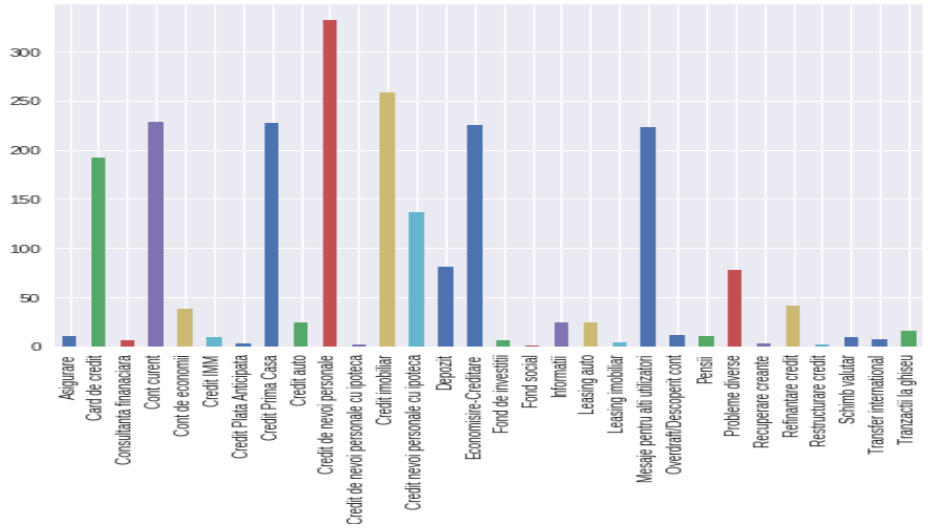

Figure 1. Financial banking products and services categories 
Data preprocessing is the initial stage in text classification which includes textual document refinements such as text document cleaning, tokenization, lower-casing, sentence segmentation, and punctuation removal in order to present the text in a clear format. [8] At this step, we have eliminated the stop words in Romanian language to reduce the size of actual data. The next step in data preprocessing is representation of text documents. We have transformed our Romanian text documents into a vector space model (VSM) in order to represent text document as a feature vector wherein each feature correspond to individual words (terms).

\subsection{Feature Selection}

The next stage of Text Classification is the selection of the most useful and valuable features for classification from the original document by keeping the words with the highest score according to a set of predefined measures. There is an extensive body of work that addresses feature selection for machine learning approaches. Most common selection methods are Chi square and document frequency, information gain and mutual information. [9][10] We applied Pearson's Chi-squared to our dataset to select the suitable features by observing if the occurrence of a specific feature is independent of the class. After selecting the most important features, each document is represented as a weighted vector based on the words found in the text. One of the widely explored approaches to enrich the feature vector representation is using n-grams (usually bigrams). We have incorporated unigrams and bigrams based on Term Frequency Inverse Document Frequency (TF-IDF) weighted method as shown in Table 1 for several financial banking products and services.

Table 1. Unigrams and bigrams of Romanian reviews

\begin{tabular}{|l|l|l|}
\hline \multicolumn{1}{|c|}{$\begin{array}{c}\text { Financial Banking } \\
\text { Product or Service }\end{array}$} & $\begin{array}{c}\text { Most correlated } \\
\text { unigrams }\end{array}$ & \multicolumn{1}{c|}{$\begin{array}{c}\text { Most correlated } \\
\text { bigrams }\end{array}$} \\
\hline Credit Prima Casa & $\begin{array}{l}\text {.prima } \\
\text {.casa }\end{array}$ & $\begin{array}{l}\text {.prima casa } \\
\text {.credit prima }\end{array}$ \\
\hline Credit de nevoi personale & $\begin{array}{l}\text {.personale } \\
\text {.nevoi }\end{array}$ & $\begin{array}{l}\text {.nevoi personale } \\
\text {.credit nevoi }\end{array}$ \\
\hline Economisire-Creditare & $\begin{array}{l}\text {.creditare } \\
\text {.economisire }\end{array}$ & $\begin{array}{l}\text {.economisire creditare } \\
\text {.locuinte economisire }\end{array}$ \\
\hline
\end{tabular}

\subsection{Supervised Machine Learning Classifiers}

Supervised classification methods, such as Support Vector Machines or Naïve Bayes have been proposed in multiple research projects [11-13]. In this paper, we have explored four supervised multi-class text classification methods shortly described in the following sub-sections.

\subsubsection{Random Forest}

Random Forest is a supervised machine learning method based on $\mathrm{K}$ decision tree as a basic categorizer. The categorization result of the Random Forest is based on an ensemble of trees and letting them vote for the most popular class using the Bagging method [14]. The bagging method is based on a combination of learning models in order to improve results where to grow each tree a random selection (without replacement) is made from the examples in the training set for each tree.

\subsubsection{Multinomial Naïve Bayes}

Nä̈ve Bayes is a probabilistic classifier often used as a baseline in text classification because it's fast and easy to implement. Naïve Bayes is based on a "naïve assumption" which assumes that all features in the document are independent of each other given the context of the class. The multinomial distribution is a unigram language model with integer word counts [17] 


\subsubsection{Linear Support Vector Machine}

Support Vector Machine (SVM) is a useful technique for sentiment classification in particular and text classification in general which belongs to a family of classifiers that perform classification by building a separating boundary between classes of interest. The SVM classifier is known as the maximum margin classifier. Support vector machines have resulted in significant success in numerous real-world learning tasks and it is considered to be the stateof-the-art in traditional machine learning.

\subsubsection{Logistic Regression}

With the wide availability of sophisticated statistical software for high-speed computers, the use of logistic regression is increasing. Logistic regression is an extension of linear regression which can be used on classification problems. Although logistic regression is used primarily with dichotomous dependent variables, the technique can be extended to situations involving outcome variables with 3 or more categories (multinomial) dependent variables. [15]

\subsection{Evaluation of Supervised Machine Learning approaches}

Cross-Validation is a statistical method of evaluating and comparing learning algorithms by dividing data into two parts: one used to learn or train a model and the other used to validate the model. In typical cross-validation, the training and validation sets must cross-over in successive rounds such that each data point has a chance of being validated against. [16] The most popular form of cross validation method is k-fold cross-validation. In k-fold crossvalidation the data is first divided into k equally sized parts or folds. For text classification the 10 -fold cross-validation $(\mathrm{k}=10)$ technique is used to evaluate or compare machine learning models in order to identify the best algorithm for the available data. Metrics such as accuracy are used to evaluate the performance of each model on each fold. For our models, we have implemented the 10-fold cross-validation technique and we measured the performance of our models using accuracy as shown in Table 2. Linear SVM is the model which obtained the highest accuracy $(91 \%)$ for multi-label text classification.

Table 2. Accuracy of supervised approaches

\begin{tabular}{|l|l|}
\hline $\begin{array}{c}\text { Supervised Machine Learning } \\
\text { algorithm }\end{array}$ & Accuracy \\
\hline Linear Support Vector Machine & 0.91 \\
\hline Logistic Regression & 0.886333 \\
\hline Multinomial Naïve Bayes & 0.858000 \\
\hline Random Forest & 0.863000 \\
\hline
\end{tabular}

\section{Performance Evaluation of multi-class text classification using Linear SVM}

To evaluate the performance of our multi-class Linear Support Vector classifier, four evaluation measures are used (recall, precision, F1-score and confusion matrix).

Precision represents the percentage of correctly classified documents among all documents that were assigned to the category by the classifier. Precision measure the exactness of a classifier and it is calculated using (1):

$$
\text { Precision }=\frac{\text { Number of documents retrieved that are relevant }}{\text { Total number of documents that are retrieved }}(1)
$$

Recall is the metric which measure the completeness, or sensitivity of a classifier. A recall for a category is defined as the fraction of correctly classified documents among all relevant documents for a category. [16] Recall is calculated using (2) 
www.conferenceie.ase.ro

$$
\text { Recall }=\frac{\text { Number of documents retrieved that are relevant }}{\text { Total number of documents that are relevant }}
$$

F1-score represents the harmonic mean of precision and recall as expressed in (3)

$$
F 1-\text { measure }=\frac{2 * \text { Precision } * \text { Recall }}{\text { Precision }+ \text { Recall }}(3)
$$

In other words, high precision means that an algorithm returned significantly more relevant results than irrelevant, also high recall means that an algorithm returned most of the relevant results. Thus, based on high values of precision and recall we can assume that the performance of our model is very good.

Confusion matrix represents one of the most informative measures of performance which a multi-class supervised learning system can rely on. Figure 2 illustrates the confusion matrix for our 30 of categories used for text classification.

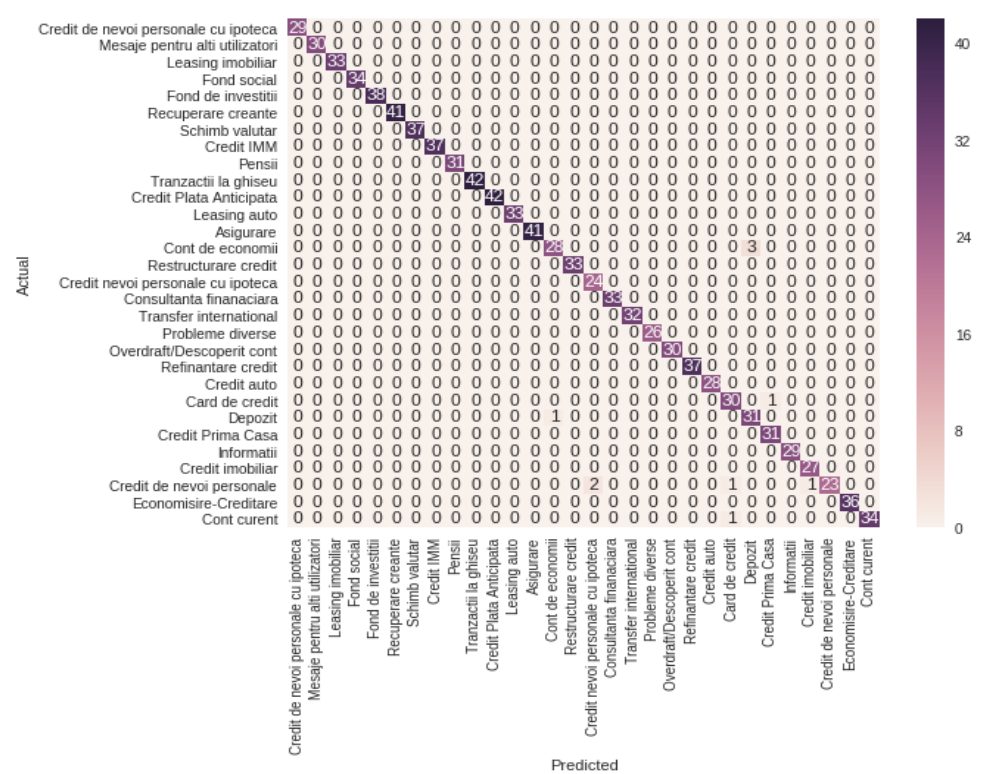

Figure 2. Confusion matrix of Support Vector Machine classification of Romanian text classification

The classification problem of reviews into financial banking products and services is difficult even for the human expert subjects. For instance, on the Romanian financial banking marketplace there are many products for real-estate loans which make it difficult for people without financial banking knowledge to understand the difference between real-estate loans. According to confusion matrix from Figure 2, the performance of our model is high with small discrepancies for some predictions. For example, one instance of "Credit de nevoi personale" is predicted as "Credit de nevoi personale cu ipoteca" or one instance of "Credit Prima Casa" is predicted as "Credit imobiliar". In the future, a new Romanian financial banking dataset will be used to validate the multi-class Linear Support Vector classifier.

\section{Conclusions and Further Research}

Nowadays, financial banking customers are exposing their comments and opinions on social media which can be shared by others also. Thus, a huge number of text documents is available online for free. The focus of this paper is on text classification research from discussion forums of Romanian financial banking marketplace. In order to conduct this research, a Romanian financial banking corpus is created via web scraping technique from Conso portal, the most popular financial banking portal from Romanian marketplace. The research work is very challenging because we deal with a small dataset and imbalanced classes. Using TF-IDF and Chi square we have investigated the efficiency of supervised machine learning methods such 
as Random Forest, Support Vector Machine, Multinomial Naïve Bayes and Logistic Regression to classify Romanian financial banking reviews. Our experimental results have shown that Support Vector Machine approach outperforms at classification of financial banking products or services. As future work, we plan to validate the classifier with a new financial banking dataset of Romanian reviews and to construct models based on neural networks for classification of Romanian reviews.

\section{References}

[1] Mofleh Al-diabat (2012), "Arabic Text Categorization Using Classification Rule Mining", Applied Mathematical Sciences, Vol. 6, 2012, no. 81, pp. 4033 - 4046.

[2] Sebastiani, F. (2002), "Machine learning in automated text categorization", ACM Publication: ACM Computing Surveys, Vol. 34, No. 1, March 2002, pp. 1-47.

[3] Aggarwal C.C., Zhai C., "A Survey of Text Classification Algorithms", in Mining Text Data. Springer, Boston, MA, 2012.

[4] A. Vasile, R. Rădulescu and I. Păvăloiu, "Topic classification in Romanian blogosphere," 12th Symposium on Neural Network Applications in Electrical Engineering (NEUREL), Belgrade, 2014, pp. 131-134.

[5] Pramokchon, P., \& Piamsa-nga, P., "A Feature Score for Classifying Class-Imbalanced Data”, Computer Science and Engineering Conference (ICSEC), 2014, pp. 409 - 414

[6] Markov, Z., \& Larose, D., T., "Data Mining the Web: Uncovering Patterns in Web Content, Structure, and Usage. New Jersey", John Wiley \& Sons Inc., 2007.

[7] Raicu, Irina, (2019), Financial Banking Dataset for Supervised Machine Learning Classification, Informatica Economica, 23, issue 1, p. 37-49

[8] Wang, Y., and Wang X.J., "A New Approach to feature selection in Text Classification", Proceedings of 4th International Conference on Machine Learning and Cybernetics, IEEE2005, Vol.6, 2005, pp. 3814-3819.

[9] P. Saengsiri, P. Meesad, S. Na Wichian and U. Herwig, "Comparison of Hybrid Feature Selection Models on Gene Expression Data," IEEE International Conference on ICT and Knowledge Engineering, 2010, pp.13 -18.

[10] C. Haruechaiyasak, W. Jitkrittum, C. Sangkeettrakarn, and C. Damrongrat, "Implementing News Article Category Browsing Based on Text Categorization Technique,", The 2008 International Conference on Web Intelligence (WI-08), 2008, pp.143-146.

[11] Thorsten Joachims. "Text categorization with support vector machines: learning with many relevant features" in Proceedings of the 10th European Conference on Machine Learning ECML-98, Chemnitz, Germany. Pages 137-142. 1998.

[12] Nello Cristianini and John Shawe-Taylor. 1999, "An Introduction to Support Vector Machines: And Other Kernel-Based Learning Methods”, Cambridge University Press, New York, NY, USA

[13] El-Kourdi, M., Bensaid, A., and Rachidi, T. "Automatic Arabic Document Categorization Based on the Naïve Bayes Algorithm", 20th International Conference on Computational Linguistics, 2004, Geneva

[14] Breiman, L., "Bagging Predictors”, Machine Learning, 26, No. 2, 123-140

[15] Chao-Ying Joanne Peng, Kuk Lida Lee \& Gary M. Ingersoll (2002), “An Introduction to Logistic Regression Analysis and Reporting", The Journal of Educational Research, 3-14

[16] Refaeilzadeh P., Tang L., Liu H. (2009) “Cross-Validation”,in: LIU L., ÖZSU M.T. (eds) Encyclopedia of Database Systems. Springer, Boston, MA

[17] Swamy, M., N., Hanumanthappa, M., \& Jyothi, N., M. (2014), "Indian Language Text Representation and Categorization using Supervised Learning Algorithm", in Intelligent Computing Applications (ICICA), 2014 International Conference on (pp. 406-410). IEEE. 